\title{
Glomerular C4d in Post-Transplant IgA Nephropathy is associated with decreased allograft survival
}

\author{
Michael Eder ${ }^{1}$. Nicolas Kozakowski ${ }^{2,3} \cdot$ Haris Omic ${ }^{1} \cdot$ Christof Aigner $^{1} \cdot$ Johannes Kläger $^{2} \cdot$ Brian Perschl $^{1}$. \\ Roman Reindl-Schwaighofer ${ }^{1}$. Gregor Bond ${ }^{1}$. Georg A. Böhmig ${ }^{1}$. Željko Kikić ${ }^{1}$
}

Received: 12 August 2020 / Accepted: 11 November 2020 / Published online: 11 December 2020

(c) The Author(s) 2020

\begin{abstract}
Background Glomerulonephritis (GN), including post-transplant IgAN (post-Tx IgAN) is an important contributor to decreased long-term allograft survival. The immunopathological detection of the complement degradation product $\mathrm{C} 4 \mathrm{~d}$ in glomeruli $(\mathrm{C} 4 \mathrm{dG})$ has been recently described as a risk factor in native kidney IgAN, however little is known about C4dG deposition in post-Tx IgAN. We hypothesized that glomerular C4d may indicate a more aggressive disease course and worse allograft survival in patients with post-Tx IgAN.

Methods In this retrospective study we assessed the presence and clinical relevance of C4dG in patients with post-transplant IgAN. We analyzed 885 renal allograft recipients, including 84 patients with post-transplant GN. All patients were transplanted between January 1999 and April 2006 and underwent at least one biopsy for differnt causes. The primary endpoint was death-censored graft survival, with a median follow-up of 9.6 (IQR 3.8-13.2) years.

Results The prevalence of post-Tx GN was $9.5 \%$. Twenty-seven patients with post-Tx IgAN were included. C4dG positive patients $(N=18,66.7 \%)$ had significantly worse allograft survival compared to C4dG negative post-Tx IgAN patients and patients without post-Tx IgAN [C4dG positive: $27.8 \%$ vs. $55.6 \%$ and $66.0 \%$; log-rank: $p=0.01]$. C4dG remained a significant risk factor (HR 2.22, 95\% CI 1.27-3.87) for allograft loss even after adjustment for T cell mediated rejection (TCMR) and antibody mediated rejection.

Conclusion Glomerular C4d deposition is an independent risk factor for worse graft-survival in patients with post-Tx IgAN, even after adjusting for other risk factors such as antibody mediated rejection. Assessment of glomerular C4d deposition may provide a valuable prognostic risk assessment tool to identify high risk patients in post-Tx IgAN.
\end{abstract}

Keywords Glomerulonephritis · IgA Nephropathy $\cdot$ Graft loss $\cdot$ Kidney allograft $\cdot$ IgAN $\cdot$ C4d $\cdot$ Glomerulus

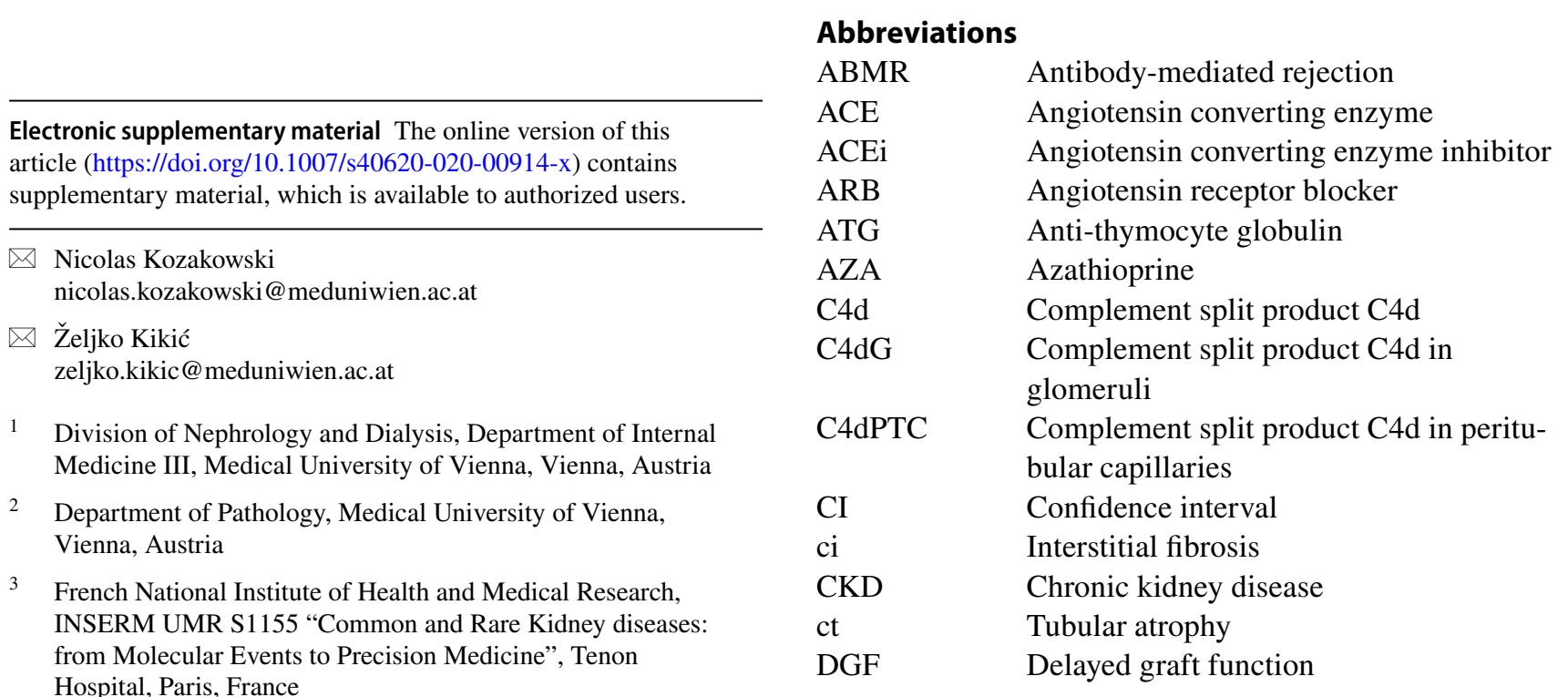




$\begin{array}{ll}\text { eGFR } & \text { Estimated glomerular filtration rate } \\ \text { ESRD } & \text { End-stage renal disease } \\ \text { Gd-IgA1 } & \text { Galactose-deficient IgA1 } \\ \text { GN } & \text { Glomerulonephritis } \\ \text { HLA } & \text { Human leukocyte antigen } \\ \text { HR } & \text { Hazard ratio } \\ \text { IAS } & \text { Immunoadsorption } \\ \text { IHC } & \text { Immunohistochemistry } \\ \text { IgAN } & \text { IgA nephropathy } \\ \text { IQR } & \text { Interquartile range } \\ \text { KM } & \text { Kaplan-Meier } \\ \text { MMF } & \text { Mycophenolate mofetil } \\ \text { post-Tx GN } & \text { Post-transplant Glomerulonephritis } \\ \text { post-Tx IgAN } & \text { Post-transplant IgA nephropathy } \\ \text { PTC } & \text { Peritubular capillaries } \\ \text { SD } & \text { Standard deviation } \\ \text { TCMR } & \text { T cell mediated rejection } \\ \text { TMA } & \text { Thrombotic microangiopathy } \\ \text { Tx } & \text { Transplantation }\end{array}$

\section{Introduction}

Chronic glomerulonephritis (GN) is one of the leading causes for the development of end-stage renal disease (ESRD) [1]. IgA nephropathy (IgAN) represents the most common primary glomerular disease and its clinical course varies from asymptomatic microhematuria to progressive chronic kidney disease (CKD) and ESRD [2-5]. Aberrant glycosylation of $\operatorname{IgA} 1$, circulating antibodies against the galactose-deficient IgA1 and the formation of $\operatorname{IgA} 1-\operatorname{IgG}$ immune complexes, are suggested mechanisms in the so called "multi-hit" hypothesis of IgAN [6-9]. In addition, emerging evidence supports an important role of complement pathways in IgAN [10-19]. Several retrospective studies suggested that glomerular $\mathrm{C} 4 \mathrm{~d}$ staining $(\mathrm{C} 4 \mathrm{dG})$ may have a prognostic value in native glomerular kidney disease [13, 20-22]. In 2012 Espinosa et al. [21] performed a retrospective multicenter analysis of 283 patients with native kidney IgAN. Around two thirds of their included patients had C4d deposits in glomerular capillaries. $\mathrm{C} 4 \mathrm{dG}$ was associated with significantly worse 20 -year renal survival (28\% vs. $85 \%$ ) and represented an independent risk factor for progression of CKD in patients with native IgAN [21]. Similar findings were observed in two subsequent analyses, independently from baseline estimated glomerular filtration rate (eGFR) $[22,23]$.

In renal transplantation, immunopathological $\mathrm{C} 4 \mathrm{~d}$ staining in peritubular capillaries (C4dPTC) is an accepted marker for the diagnosis of antibody-mediated rejection (ABMR) [24]. Linear C4d deposits in PTC are considered to be induced by HLA-antibodies with subsequent activation of the classical pathway and are associated with a higher risk of allograft loss [25-27]. In native and post-transplant IgA Nephropathy (post-Tx IgAN) similar pathomechanisms have been suggested [28-30], but in contrast to native kidney IgAN, the role of $\mathrm{C} 4 \mathrm{dG}$ in post-transplant IgAN is yet to be determined. Further, evidence regarding the frequency and clinical significance of $\mathrm{C} 4 \mathrm{dG}$ depositions in post-transplant GN is scarce [31]. We hypothesize that similar to reports in native kidney IgAN [32], C4dG deposits in post-Tx IgAN may indicate worse clinical outcome. Aim of our study was therefore to analyze 1) the frequency of $\mathrm{C} 4 \mathrm{dG}$ deposition in allograft recipients with recurrent or de-novo IgAN, 2) the long-term death-censored graft survival in $\mathrm{C} 4 \mathrm{dG}$ positive post-Tx IgAN patients and 3) their clinical course compared to $\mathrm{C} 4 \mathrm{dG}$ negative patients.

\section{Methods}

\section{Study design and patients}

The present study was designed as a retrospective singlecenter analysis. The objective was to assess the presence and clinical relevance of $\mathrm{C} 4 \mathrm{dG}$ staining in patients with de-novo or recurrent post-transplant IgA nephropathy. The primary outcome was death-censored graft-survival, defined as date of initiation of any renal replacement therapy. We analyzed all patients with consecutive renal transplantation between January 1st 1999 and April 1st $2006(N=1248)$ and available biopsy results as well as clinical follow-up until January 1st 2017. All biopsies were for cause biopsies, performed upon unexplainable graft dysfunction and/or proteinuria. Study approval was obtained from the local institutional ethics committee (EK-number 1490/2017). All procedures were conducted according to the Declarations of Helsinki and Istanbul.

\section{Baseline characteristics}

Eight hundred and eighty-five patients underwent allograft biopsy for various causes and were included into the study. Baseline characteristics were analyzed from digital patient records and included relevant demographic and transplantassociated findings as well as the occurrence of early (within the first six months after transplant) rejections including $\mathrm{T}$ cell mediated rejection (TCMR), ABMR and Borderline lesions and eGFR and proteinuria after one year. The glomerular filtration rate was estimated using the Mayo Clinic formula [33].

\section{Analysis of biopsies and C4d staining}

Of the 885 included patients, a diagnosis of post-transplant glomerulonephritis was made in $84(9.5 \%)$ of them. 
Individuals with suspected post-Tx GN underwent detailed immunohistochemistry and ultrastructural analysis with electron microscopy (if material was available). Details on staining procedures and electron microscopy are provided as a supplemental section. The diagnosis of post-Tx IgAN was defined by the presence of glomerular IgA-dominant or codominant immune deposits assessed by immunohistochemistry or immunofluorescence, as described before [34]. In the literature the definition of $\mathrm{C} 4 \mathrm{dG}$ in native kidney $\operatorname{IgAN}$ is variable, depending on the staining technique (IF versus IHC) and includes the mesangium and glomerular capillary walls $[14,16,17,19]$. Therefore, $\mathrm{C} 4 \mathrm{dG}$ deposition was defined as an IHC-based detection of granular C4d deposits in $>5 \%$ of peripheral glomerular capillaries or $\mathrm{C} 4 \mathrm{~d}$ deposits in the mesangium (Fig. 1). All included biopsies with post-Tx GN were retrospectively re-analyzed by two nephropathologists (N.K., J.K.) blinded to clinical data. Biopsies were reassessed according to the 2017 Banff scheme [35]. C4d positive ABMR was considered present if linear, circumferential C4d deposits in PTC were above 0\% [36]. Medullary vasa recta were also assessed if available. The diagnosis of C4d negative ABMR had not been established during the observation period for most patients. To accommodate the potential effect of $\mathrm{C} 4 \mathrm{~d}$ negative ABMR on graft survival, and due to the lack of post-transplant HLA antibody results (which were not standard of care at our center during the study period), we chose a broader definition of the histomorphology compatible for $\mathrm{C} 4 \mathrm{~d}$ negative $\mathrm{ABMR}$ (= suspicion of $\mathrm{C} 4 \mathrm{~d}$ negative $\mathrm{ABMR}$ ), which consisted of the presence of inflammatory lesions compatible with ABMR in at least two different renal compartments including a $\mathrm{g}$ score $>0$, a ptc score $>0$, post-transplant thrombotic microangiopathy (TMA), V3 lesions and a cg score $>0$. Additionally, following the BANFF guidelines, a ptc score $>0$ was not considered sufficient for ABMR diagnosis in the presence of tubulointerstitial infiltrates. Second, g and cg lesions were not scored in the presence of glomerulonephritis. The Oxford criteria (MEST-C score) were applied according to the 2017 published consensus reports [37].

\section{Treatment}

The treatment policy for post-Tx IgAN during the respective time period was watchful waiting and the introduction of angiotensin converting enzyme inhibitor (ACEi)/angiotensin receptor blocker (ARB) (if not already established) in the majority of cases. Only in cases with rapid deterioration of graft function intensified immunosuppressive regimens were applied. Those regimens were: steroid bolus therapy in three patients, switch to calcineurin inhibitors and/or steroid bolus therapy in four patients and one patient received oral cyclophosphamide ( $2 \mathrm{mg}$ per kilogram). All rejection episodes were treated as follows: patients with TCMR Banff $\geq$ IA-IIA or Banff Borderline lesions with concomitant allograft dysfunction received a steroid pulse. Steroidrefractory or Banff IIB rejections were treated with antithymocyte globulin (ATG) over a course of ten to fourteen days. C4d positive ABMR with allograft dysfunction was treated in the majority of cases as previously described [38].

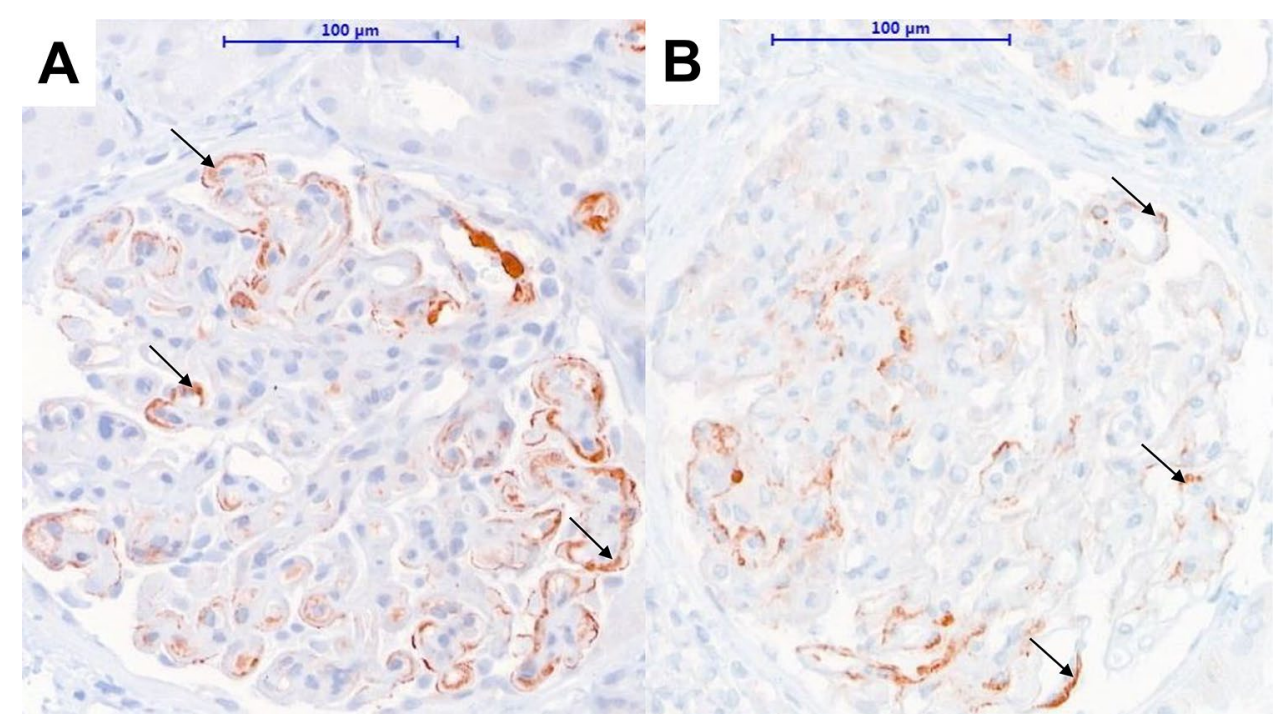

Fig. 1 Immunohistochemical staining for C4d deposits. Representative examples of $\mathrm{C} 4 \mathrm{~d}$ positive post-Tx IgAN patients. Both biopsies show $\mathrm{C} 4 \mathrm{~d}$ deposits in glomerular peripheral capillary walls. In the first case (a), the patient was diagnosed with recurrence of $\operatorname{IgAN} 8$ years after transplantation and lost his allograft within the follow- ing 2 years. In (B), the primary underlying renal disease was a not further characterized glomerulonephritis. The patient was diagnosed with post-Tx IgAN 7 years after transplantation and lost his allograft 15 months later. Black arrows indicate granular glomerular $\mathrm{C} 4 \mathrm{~d}$ in peripheral capillary loops. Magnification scale 400X 


\section{Statistical analysis}

Continuous variables were expressed as mean and standard deviation or median and interquartile range, whichever appropriate. Categorical variables were expressed as absolute and relative frequencies. Unpaired Student's $t$ or Mann-Whitney-U was used for comparison of continuous data; group comparisons of categorical variables were analyzed by Fisher's exact or Chi Squared $\left(\chi^{2}\right)$ test. Kaplan-Meier analysis and Mantel-Cox log-rank test were used for comparison of graft survival. Graft survival was compared between 1) patients with and without post-Tx GN and 2) between $\mathrm{C} 4 \mathrm{dG}$ positive and negative post-Tx IgAN patients and patients without post-Tx GN (control group). For Cox regression analysis, variables differing between the groups with GN and without GN or significantly related to the primary endpoint were included. Variables with $p<0.05$ in the univariate model were included into the multivariate regression analysis. $p$-values $<0.05$ were considered statistically significant. Statistical analysis and graph design were performed using commercially available software systems (Microsoft Office Excel; Microsoft Corp., Redmond, WA; SPSS; Version 25, SPSS Inc., Chicago, IL, GraphPad Prism version 8.40, GraphPad Software, La Jolla, CA, USA).

\section{Results}

\section{Study cohort}

In total 885 patients with indication biopsy and a median follow-up of 9.6/3.8-13.2 years (median/IQR) were included. Most patients were male (63.5\%), mean donor and recipient age were $48.7 \pm 15.0$ and $50.9 \pm 13.7$ years, respectively. One hundred and two (11.5\%) patients received transplants from living donors. Most patients $(N=656 / 74.3 \%)$ were on Cyclosporin A-based baseline immunosuppression. Further baseline parameters are shown in Table 1.

\section{Patients with post-Tx GN}

Biopsy confirmed post-transplant glomerulonephritis was diagnosed in 84 patients within 3/1-6 (median/IQR) years after transplantation, representing a prevalence of $9.5 \%$. Post-Tx IgAN was found in 34 (40.5\%) patients. Patients with post-Tx GN were significantly younger $(46.9 \pm 13.9$ vs. $51.4 \pm 13.6$ years; $p=0.008)$ at transplantation and had more often received a prior allograft $(33.3 \%$ vs. $16.7 \%$ in patients without $\mathrm{GN}, p<0.001)$. Glomerulonephritis was the major cause for end-stage renal disease in the post-Tx GN group ( $44.0 \%$ vs. $20.3 \%$ in patients without post-Tx GN, $p<0.001$, Supplemental Table 1). Recurrence of primary glomerular disease was assumed in eighteen $(21.4 \%)$ patients. There were no patients with IgA vasculitis (Purpura-SchönleinHenoch) as native kidney disease.

\section{Post-transplant Glomerulonephritis is associated with graft survival}

Figure 2 shows KM graft survival of patients with post-Tx GN compared to all patients without post-Tx GN. Deathcensored graft survival was significantly worse in the postTx GN group $(36.1 \%$ vs $66 \%$; log-rank test $p<0.001)$. Univariate Cox-regression revealed a two-fold increase in the risk for graft loss in patients with post-Tx GN [HR 2.1; (95\% CI 1.4-3.2); $p<0.001]$.

\section{Post-transplant IgA nephropathy (post-Tx IgAN)}

Thirty-four patients $(26.7 \%$ female) were diagnosed with post-Tx IgAN within 3.3/1.4-7.1 years (median/IQR) after transplantation. Recurrence of disease was suspected in nine $(26.5 \%)$ patients and was significantly more frequent in living donor recipients [57.1\% (4/7) vs. $18.5 \%(5 / 27)$ in deceased donor recipients, $\mathrm{p}=0.039]$. The risk for recurrence of IgAN was not associated with pre-sensitization or early $\mathrm{C} 4 \mathrm{~d}$ negative ABMR. None of the included patients received a steroid-sparing immunosuppressive regimen. Serum creatinine at the time of index biopsy was 2.0/1.7-2.6 mg/dl (median/IQR) and did not significantly differ from other post-Tx GN patients [2.0/1.6-2.7 mg/dl (median/IQR), $p=0.26]$. Twenty $(66.7 \%)$ patients were on ACE-inhibitors or ARBs. Further baseline characteristics are shown in Table 2. KM death-censored graft survival was significantly worse in patients with post-Tx IgAN compared to patients without post-Tx GN [38.2\% vs. $66.0 \%$, log-rank test $p=0.004$, univariate Cox-regression: HR $2.2 ;(95 \% \mathrm{CI}$ $1.5-4.9) ; p=0.001]$. Death-censored graft survival was not significantly different between de-novo or recurrent IgAN (log-rank test, $p=0.93$ ).

\section{Immunohistochemistry in post-Tx IgAN patients}

C4dG stainings were available in 27 patients with post-Tx IgAN. Of those, 18 (66.7\%) showed positive $\mathrm{C} 4 \mathrm{dG}$ staining. In most cases $(N=16)$, granular $\mathrm{C} 4 \mathrm{dG}$ was found in peripheral capillary loops. Four patients had mesangial $\mathrm{C} 4 \mathrm{~d}$ deposits (two of them had mesangial and peripheral capillary C4d deposits). Glomerular basal membrane splitting was found in five out of eleven patients with available silver staining and peripheral capillary $\mathrm{C} 4 \mathrm{~d}$ deposits. In three biopsies concomitant C4d positive ABMR (linear C4d positive in PTC) was diagnosed. Those patients were diagnosed with chronic antibody-mediated rejection. Simultaneous tubulointerstitial infiltrates were observed in fourteen patients (BANFF $\geq 1 N=7$, BANFF Borderline $N=7$ ). Neither the 
Table 1 Baseline findings of included patients

\begin{tabular}{|c|c|c|c|c|}
\hline \multirow[t]{2}{*}{ Parameters } & \multirow[t]{2}{*}{ All patients $(N=885)$} & \multicolumn{2}{|c|}{ Glomerulonephritis in biopsy } & \multirow[t]{2}{*}{$P$ value } \\
\hline & & No GN $(N=801)$ & $\mathrm{GN}(N=84)$ & \\
\hline Female gender, $N(\%)$ & $323(36.5)$ & $293(36.6)$ & $30(35.7)$ & 0.88 \\
\hline Recipient age, mean (SD) & $50.9(13.7)$ & $51.4(13.6)$ & $46.9(13.9)$ & 0.008 \\
\hline Donor age, mean (SD) & $48.7(15.0)$ & $48.9(15.0)$ & $46.8(15.0)$ & 0.16 \\
\hline Living donor, $N(\%)$ & $102(11.5)$ & $88(11.0)$ & $14(16.7)$ & 0.12 \\
\hline HLA mismatch (A, B, DR), median (IQR) & $3(2-4)$ & $3(2-4)$ & $3(2-3)$ & 0.28 \\
\hline Years of follow-Up, median (IQR) & $9.6(3.8-13.2)$ & $9.9(3.6-13.3)$ & $8.3(4.7-11.5)$ & 0.52 \\
\hline eGFR 1 year after Tx, $\mathrm{ml} / \mathrm{min} / 1.73 \mathrm{~m}^{2}$, median (IQR) & $53(33-75)$ & $52(34-70)$ & $53(33-75)$ & 0.060 \\
\hline Protein/creatinine ratio 1 year after $\mathrm{Tx}, \mathrm{mg} / \mathrm{g}$, median (IQR) & $77(0-237)$ & $70(0-235)$ & $104(0-312)$ & 0.26 \\
\hline Re-transplantation, $N(\%)$ & $162(18.3)$ & $134(16.7)$ & $28(33.3)$ & $<0.001$ \\
\hline Cold ischemia time in hours, median (IQR) & $13(8-19)$ & $13(8-19)$ & $11(6-16)$ & 0.010 \\
\hline DGF, $N(\%)$ & $194(22.3)$ & $178(22.7)$ & $16(19.0)$ & 0.45 \\
\hline \multicolumn{5}{|l|}{ Immunosuppression } \\
\hline Initially Tacrolimus, $N(\%)$ & $148(16.8)$ & $135(16.9)$ & $13(15.7)$ & 0.78 \\
\hline Initially Ciclosporin, $N(\%)$ & $656(74.3)$ & $590(73.8)$ & $66(79.5)$ & 0.25 \\
\hline Initially MMF, $N(\%)$ & $827(94.1)$ & $750(94.2)$ & $77(92.8)$ & 0.59 \\
\hline Initially AZA, $N(\%)$ & $14(1.6)$ & $13(1.6)$ & $1(1.2)$ & 0.77 \\
\hline \multicolumn{5}{|l|}{ Rejections } \\
\hline Early C4d pos. ABMR, $N(\%)$ & $105(11.9)$ & $95(11.9)$ & $10(12)$ & 0.96 \\
\hline Early C4d neg. ABMR*, $N(\%)$ & $77(8.7)$ & $59(7.4)$ & $18(21.4)$ & $<0.001$ \\
\hline Early Banff Borderline, $N(\%)$ & $133(15.0)$ & $125(15.6)$ & $8(9.5)$ & 0.14 \\
\hline Early TCMR Banff $\geq 1, N(\%)$ & $270(30.5)$ & $246(30.7)$ & $24(28.6)$ & 0.69 \\
\hline \multicolumn{5}{|l|}{ Underlying renal disease } \\
\hline Diabetic nephropathy, $N(\%)$ & $103(11.6)$ & $100(12.5)$ & $3(3.6)$ & 0.015 \\
\hline Cystic kidney disease, $N(\%)$ & $101(11.4)$ & $92(11.5)$ & $9(10.7)$ & 0.83 \\
\hline Glomerulonephritis, $N(\%)$ & $200(22.6)$ & $163(20.3)$ & $37(44.0)$ & $<0.001$ \\
\hline IgA-nephropathy, $N(\%)$ & $51(5.8)$ & $41(5.1)$ & $10(11.9)$ & 0.011 \\
\hline Vascular nephropathy, $N(\%)$ & $54(6.1)$ & $52(6.5)$ & $2(2.4)$ & 0.14 \\
\hline Chronic pyelonephritis, $N(\%)$ & $45(5.1)$ & $40(5.0)$ & $5(6.0)$ & 0.70 \\
\hline Obstructive nephropathy, $N(\%)$ & $19(2.1)$ & $18(2.2)$ & $1(1.2)$ & 0.53 \\
\hline Other, $N(\%)$ & $97(11.0)$ & 87 (10.9) & $10(11.9)$ & 0.77 \\
\hline Unknown, $N(\%)$ & $266(30.1)$ & $249(31.1)$ & $17(20.2)$ & 0.039 \\
\hline
\end{tabular}

$A B M R$ antibody-mediated rejection, $A Z A$ azathioprine, $D G F$ delayed graft function, $G N$ glomerulonephritis, $I Q R$ interquartile range; $M M F$ mycophenolate mofetil; $N$ number, Neg. negative, $S D$ standard deviation, TCMR T cell mediated rejection, $T x$ transplantation, Pos. positive. Mayo Clinic estimation was used to calculate the eGFR. Early rejection was classified as occurrence within the first six months after transplantation; *Suspicion of C4d negative ABMR, which consisted of the presence of at least two lesions being compatible with ABMR including a $\mathrm{g}$ score $>0$, a ptc score $>0$, post-transplant thrombotic microangiopathy (TMA), V3 lesions and a cg score $>0$

Oxford MEST-C single scores nor the sum score differed significantly between $\mathrm{C} 4 \mathrm{dG}$ positive- and negative patients (data not shown). Crescents were found in two patients with post-Tx IgAN. As shown in Table 3, neither baseline variables nor laboratory findings at the time of index biopsy, including graft function and proteinuria, differed significantly between $\mathrm{C} 4 \mathrm{dG}$ positive and negative post-Tx IgAN patients. Moreover, intensified immunosuppression as a treatment of post-Tx IgAN was rare and did not differ between both groups (data not shown). C4dG positivity was a constant finding in follow-up biopsies (available in six $\mathrm{C} 4 \mathrm{dG}$ positive patients). All patients had positive $\mathrm{C} 4 \mathrm{dG}$ stainings in follow-up biopsies (three with mesangial and three patients with peripheral capillary C4d deposits).

\section{C4dG is associated with worse death-censored graft survival}

Kaplan Meier analysis revealed a significantly worse deathcensored allograft survival of C4dG positive post-Tx IgAN patients [death-censored graft-survival: $\mathrm{C} 4 \mathrm{dG} 27.8 \%$ vs. $55.6 \%$ in C4dG negative post-Tx IgAN patients and $66.0 \%$ in patients without GN; log-rank: $p=0.01$ ] (Fig. 3). Exclusion of the three patients with concomitant $\mathrm{C} 4 \mathrm{~d}$ positive ABMR 
Fig. 2 Kaplan-Meier allograft survival in relation to the presence of post-Tx GN. Groups included were patients with glomerulonephritis after transplantation $(N=84$, black solid line) and patients without glomerulonephritis after renal transplantation $(N=801$, grey solid line). Log-Rank test revealed significant, worse allograft survival in patients with post-Tx GN $(p<0.001)$

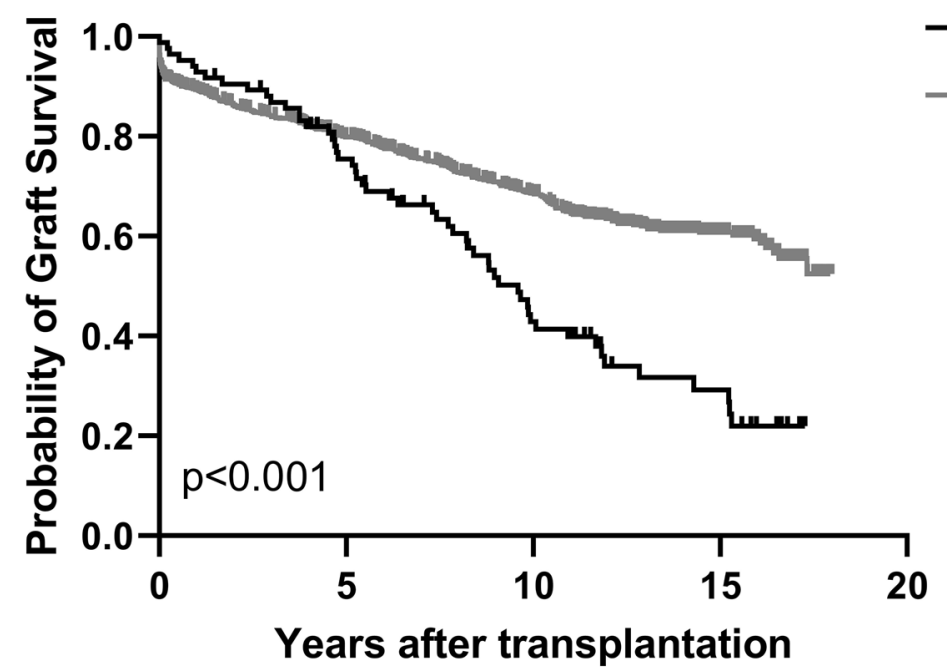

- Post-Tx GN

Patients at risk

$\begin{array}{llll}84 & 59 & 30 & 13 \\ 801 & 552 & 396 & 121\end{array}$

did not materially change the highly significant association of $\mathrm{C} 4 \mathrm{dG}$ with graft loss (data not shown). In univariate Cox-regression analysis, $\mathrm{C} 4 \mathrm{dG}$ positive $\mathrm{IgAN}$ represented a highly significant risk factor for allograft loss [HR $2.22(95 \%$ CI $1.27-3.87), p=0.005]$. C4dG positive post-Tx IgAN remained independently associated with death-censored graft-loss (HR 3.57, 95\% CI 1.80-7.09, $p=0.001$ ) even after adjustment for multiple baseline covariates including ABMR, Banff Borderline lesion and TCMR (Table 4).

\section{Discussion}

In this analysis of a representative and well characterized cohort of renal transplant recipients, we demonstrated the clinical relevance of glomerular capillary $\mathrm{C} 4 \mathrm{~d}$ staining as a profound prognostic marker in patients with post-Tx IgA nephropathy. To the best of our knowledge, this is the first study showing that post-Tx IgAN patients with C4dG deposits had significantly worse death-censored allograft survival. The substantially increased risk for allograft loss remained significant even after adjustment for other known risk factors such as HLA-mismatch, donor age and the occurrence of biopsy-proven rejection. The prognostic impact of $\mathrm{C} 4 \mathrm{dG}$ in post-Tx IgAN is in line with descriptions of patients with native kidney $\operatorname{IgAN}$, where $\mathrm{C} 4 \mathrm{dG}$ depositions have been associated with an overall worse renal prognosis [15, 21, $22,39]$. However, in renal transplant cohorts with posttransplant glomerulonephritis the interpretation of immunopathological $\mathrm{C} 4 \mathrm{dG}$ may be hampered due to HLA-antibody-triggered $\mathrm{C} 4 \mathrm{dPTC}$ and/or C4dG. In our cohort only three subjects were diagnosed with concomitant ABMR, we can therefore safely exclude ABMR as a driving force of increased risk for graft loss in post-Tx IgAN patients. Nevertheless, the underlying pathomechanisms are still not fully understood. It was hypothesized that more progressive forms of IgAN are associated with endocapillary hypercellularity and vascular injuries, leading to complement activation and deposits of complement degradation products in various glomerular compartments-in particular in mesangial cells and glomerular capillary walls [40]. Recent studies in native kidney $\operatorname{IgAN}$ however have suggested additional clinical relevance of $\mathrm{C} 4 \mathrm{~d}$ staining in other renal compartments including tubules, interlobular arteries and arterioles [14, 16, 17, 19].

Graft loss occurred in two thirds of our group of $\mathrm{C} 4 \mathrm{dG}$ positive post-Tx IgAN patients, within a median of two years after the index biopsy. At the time of index biopsy, neither graft function nor proteinuria differed between $\mathrm{C} 4 \mathrm{~d}$ positive- and negative IgAN patients. Based on our findings, we therefore hypothesize that $\mathrm{C} 4 \mathrm{dG}$ may help to identify a progressive course of disease in patients with post-Tx IgAN, even before a severe deterioration of graft function is observed. This is in contrast to studies in native kidney IgAN [21, 39]. In 2014, Espinosa et al. [21] reported on 283 patients with IgAN (38.5\% C4d positive). In their study, patients with $\mathrm{C} 4 \mathrm{dG}$ positive biopsies already had significantly more proteinuria and worse eGFR at the time of index biopsy. We believe that the difference in our findings may be due to regular follow-ups of transplant recipients and thus earlier diagnosis of post-Tx IgAN compared to native kidney IgAN patients with very heterogeneous disease courses. Nevertheless, in both studies the association of $\mathrm{C} 4 \mathrm{dG}$ with ESRD remained statistically significant even after correction 
Table 2 Characteristics of all 34 post-Tx IgAN patients

\begin{tabular}{ll}
\hline Parameters & $\begin{array}{l}\text { Patients with } \\
\text { post-Tx IgAN } \\
(N=34)\end{array}$ \\
\hline Female gender, $N(\%)$ & $9(26.7)$ \\
Donor age, median (IQR) & $49(37-56)$ \\
Age at transplantation, median (IQR) & $48(32-57)$ \\
HLA-mismatch, median (IQR) & $3(2-3)$ \\
Living donor transplantation, $N(\%)$ & $7(20.6)$ \\
First transplantation, $N$ (\%) & $27(79.4)$ \\
Second transplantation, $N(\%)$ & $6(17.6)$ \\
Recurrence of disease, $N$ (\%) & $9(26.5)$ \\
Histological findings in index biopsies & \\
Interstitial Inflammation (i), median (IQR) & $1(0-1)$ \\
Tubulitis (t), median (IQR) & $1.5(0-2)$ \\
Intimal arteritis (v), median (IQR) & $0(0-0)$ \\
Arteriolar hyalinosis (ah), median (IQR) & $1(0-2.8)$ \\
Interstitial fibrosis (ci), median (IQR) & $2(1-2)$ \\
Tubular atrophy (ct), median (IQR) & $1(1-2)$ \\
Mesangial matrix expansion (mm), median (IQR) & $2(1-2)$ \\
Active crescents, $N$ (\%) & $2(5.9)$ \\
Renal parameters at the time of biopsy & \\
Creatinine at biopsy, mg/dl, median (IQR) & $2.0(1.7-2.6)$ \\
Protein/creatinine ratio mg/g, median (IQR) & $2.2(0.6-4.6)$ \\
eGFR ml/min/1.73 m ${ }^{2}$, median (IQR) & $37.1(23.7-48.6)$ \\
Creatinine 1 year after biopsy, mg/dl, median & $2.4(1.6-4.1)$ \\
(IQR) & \\
Years after transplantation, median (IQR) & $3.3(1.4-7.1)$ \\
Years of follow-up duration, median (IQR) & $9.4(5.6-11.8)$ \\
\hline
\end{tabular}

$e G F R$ estimated glomerular filtration rate, HLA human leukocyte antigen, $I Q R$ interquartile range, $N$ number, Post-Tx IgAN post-transplant IgA nephropathy, Mayo Clinic estimation was used to calculate the eGFR

for baseline eGFR. In contrast, in our study $\mathrm{C} 4 \mathrm{dG}$ negative patients had death-censored graft survival that was comparable to the control group, suggesting an acceptable allograft prognosis even after post-Tx IgAN diagnosis. Most of our patients were not subjected to intensified immunosuppression after the diagnosis of post-Tx IgAN. Moreover, concomitant TCMR and Banff Borderline lesions were routinely treated with steroid boli, and their prevalence was not different between the two groups (data not shown). We can therefore safely exclude a relevant treatment bias between $\mathrm{C} 4 \mathrm{dG}$ positive and negative groups.

Another finding that needs to be emphasized is that posttransplant glomerulonephritis was a major contributor for decreased long-term graft survival. Not only post-Tx IgAN patients but all patients with a diagnosed post-Tx GN had significantly worse death-censored graft-survival compared to the control group. This is in line with previously published findings from Chailimpamontree et al. [41].
One major strength of this study is the long followup period. We believe this is essential because post-Tx GNs often manifest late and show a heterogeneous disease course. Consequently, insufficient follow-up periods may lead to an underestimation of the decreased long-term survival of post-Tx GNs. Further, multiple relevant risk factors for decreased allograft survival, including donorassociated risk factors, were available and were included into the multivariate analysis.

Our study has limitations that need to be discussed. First, though the study design was retrospective, we have included a large sample of post-transplant biopsies with long-term follow-up and corrected our findings for multiple covariates including HLA-mismatch, and early- and concomitant rejections. Moreover, the follow-up period of included biopsies was comparable between $\mathrm{C} 4 \mathrm{dG}$ positive and negative patients, arguing against a relevant time selection bias. We found predominantly peripheral capillary- rather than mesangial C4d positivity, a deposition pattern that may seem counterintuitive at first glance. Nevertheless, our findings are in concordance with previous reports from patients with native $\mathrm{Ig} \mathrm{AN}$, where $\mathrm{C} 4 \mathrm{~d}$ was described in various glomerular compartments: Rath et al. reported that $40 \%$ of his patients with IgAN showed $\mathrm{C} 4 \mathrm{~d}$ depositions in the glomerular capillary walls [17]. These findings were in line with a report from Meng et al. [16] where $\mathrm{C} 4 \mathrm{~d}$ was found in the mesangium as well as in capillary loops. Differences in IgA and C4d staining patterns may be related to possible alterations of non-endothelial C4d deposits resulting from formalin-fixation or paraffinembedding [42]. Moreover, despite equally distributed mesangial- and peripheral $\mathrm{C} 1 \mathrm{q}$ deposition, a potential role of MBL pathway activation cannot be excluded in certain patients. Another important limitation is the lack of systematic post-transplant assessment of anti HLA- antibodies. We have corrected this by using a broad definition of histological features compatible with $\mathrm{C} 4 \mathrm{~d}$ negative $\mathrm{ABMR}$, and by the inclusion of both $\mathrm{C} 4 \mathrm{~d}$ positive and $\mathrm{C} 4 \mathrm{~d}$ negative ABMR in multivariate models. However, we cannot completely rule out the possibility that some biopsies were not attributed to ABMR.

Further, it is important to know that isolated microhematuria after transplantation was not considered a biopsy indication in the study period.

In conclusion, this study shows a prognostic value of C4dG deposition in patients with post-transplant IgAN. $\mathrm{C} 4 \mathrm{dG}$ positivity was significantly associated with graft loss and remained an independent risk factor even after correction for covariates in the multivariate analysis. These findings are of clinical importance providing new evidence and suggesting that $\mathrm{C} 4 \mathrm{dG}$ assement may be useful for a riskbased management of post-Tx IgAN patients. 
Table 3 Patient characteristics in relation to the presence of glomerular C4d

\begin{tabular}{|c|c|c|c|c|}
\hline \multirow[t]{2}{*}{ Parameters } & \multirow{2}{*}{$\begin{array}{l}\text { All patients } \\
(N=27)\end{array}$} & \multicolumn{2}{|c|}{$\mathrm{C} 4 \mathrm{dG}$ in patients with post-Tx IgAN } & \multirow[t]{2}{*}{$p$ value } \\
\hline & & Positive $(N=18)$ & Negative $(N=9)$ & \\
\hline Donor age, median (IQR) & $49(37-57)$ & $47(36-57)$ & $51(46-55)$ & 0.31 \\
\hline Age at transplantation, median (IQR) & $51(39-58)$ & $50(32-56)$ & $53(47-58)$ & 0.28 \\
\hline HLA-mismatch, median (IQR) & $3(2-4)$ & $3(2-4)$ & $3(2-3)$ & 0.71 \\
\hline \multicolumn{5}{|l|}{ Rejections } \\
\hline Early C4d pos. ABMR, $N(\%)$ & $3(11.1)$ & $2(11.1)$ & $1(11.1)$ & $>0.99$ \\
\hline Early C4d neg. ABMR*, $N(\%)$ & $8(29.6)$ & $7(38.9)$ & $1(11.1)$ & 0.14 \\
\hline Early Banff Borderline, $N(\%)$ & $1(3.7)$ & $1(5.6)$ & 0 & 0.47 \\
\hline Early TCMR Banff $\geq 1 ; N(\%)$ & $9(33.3)$ & $7(38.9)$ & $2(22.2)$ & 0.39 \\
\hline \multicolumn{5}{|l|}{ Findings at the time of index biopsy } \\
\hline Years after transplantation, median (IQR) & $3(1.6-6.8)$ & $2.9(1.6-6.8)$ & $3.7(2.3-5.3)$ & 0.940 \\
\hline Creatinine at biopsy, mg/dl, median (IQR) & $2.0(1.7-2.6)$ & $2.1(1.7-2.7)$ & $2.0(1.9-2.6)$ & 0.93 \\
\hline eGFR 1 year after biopsy, ml/min/1.73 m², median (IQR) & $35.6(23.3-45.5)$ & $33.4(23.8-50.5)$ & $37.0(22.6-40.0)$ & 0.59 \\
\hline Protein/creatinine ratio mg/g, median (IQR) & $2100(700-4300)$ & $3300(400-4600)$ & $2100(1200-2200)$ & $>0.99$ \\
\hline ACEi/ARB at biopsy, $N(\%)$ & $16 / 23(69.6)$ & $11 / 15(73.3)$ & $5 / 8(62.5)$ & 0.59 \\
\hline Number of antihypertensive drugs, median (IQR) & $3(3-4)$ & $3(3-4)$ & $3(3-4)$ & 0.59 \\
\hline More than three antihypertensives, $N(\%)$ & $9 / 23(39)$ & $7 / 15(46.7)$ & $2 / 8(25)$ & 0.31 \\
\hline \multicolumn{5}{|l|}{ Oxford MEST-C } \\
\hline MEST-C sum score, median (IQR) & $1(0.3-2)$ & $1(0.3-2)$ & $1(0.8-1.3)$ & 0.78 \\
\hline \multicolumn{5}{|l|}{ Immunohistochemistry findings } \\
\hline IgM mesangial, $N(\%)$ & $27(100)$ & $18(100)$ & $9(100)$ & N/A \\
\hline IgM peripheral, $N(\%)$ & $12(44.4)$ & $8(44.4)$ & $4(44.4)$ & $>0.99$ \\
\hline C3 mesangial, $N(\%)$ & $18(66.7)$ & $11(61.1)$ & $7(77.8)$ & 0.39 \\
\hline $\mathrm{C} 3$ peripheral, $N(\%)$ & $5(18.5)$ & $4(22.2)$ & $1(11.1)$ & 0.48 \\
\hline C1q mesangial, $N(\%)$ & $26(96.3)$ & $17(94.4)$ & $9(100)$ & 0.47 \\
\hline 1q peripheral, $N(\%)$ & $11(40.7)$ & $8(44.4)$ & $3(33.3)$ & 0.58 \\
\hline \multicolumn{5}{|l|}{ Outcomes } \\
\hline Follow-Up time in months, median (IQR) & $103.2(81.1-119.1)$ & $98.9(75.2-118)$ & $103.6(82.6-141.6)$ & 0.43 \\
\hline Death during follow-up, $N(\%)$ & $11(40.7)$ & $8(44.4)$ & $3(33.3)$ & 0.58 \\
\hline \multicolumn{5}{|l|}{ Underlying renal disease } \\
\hline Glomerulonephritis, $N(\%)$ & $12(44.4)$ & $8(44.4)$ & $4(44.4)$ & $>0.99$ \\
\hline IgA Nephropathy, $N(\%)$ & $8(29.6)$ & $4(22.2)$ & $4(44.4)$ & 0.23 \\
\hline Diabetic Nephropathy, $N(\%)$ & $1(3.7)$ & $1(5.6)$ & 0 & 0.47 \\
\hline Vascular Nephropathy, $N(\%)$ & $1(3.7)$ & $1(5.6)$ & 0 & 0.47 \\
\hline Cystic kidney disease, $N(\%)$ & $2(7.4)$ & $2(11.1)$ & 0 & 0.30 \\
\hline Unknown, $N(\%)$ & $7(25.9)$ & $5(27.8)$ & $2(22.2)$ & 0.76 \\
\hline
\end{tabular}

$A B M R$ antibody-mediated rejection, $A C E i$ angiotensin converting enzyme inhibitor, $A R B$ Angiotensin receptor blocker, $e G F R$ estimated glomerular filtration rate, $H L A$ human leukocyte antigen, $I Q R$ interquartile range, $N$ number, neg. negative, pos. positive, Post-Tx IgAN post-transplant IgA nephropathy, TCMR T-cell mediated rejection. Early rejection was classified as occurrence within the first 6 months after transplantation; the antihypertensive treatment was known in 23 patients; Mayo Clinic estimation was used to calculate the eGFR; *: Suspicion of C4d negative ABMR, which consisted of the presence of at least two lesions being compatible with ABMR including a g score $>0$, a ptc score $>0$, post-transplant thrombotic microangiopathy (TMA), V3 lesions and a cg score $>0$ 
Fig. 3 Kaplan-Meier allograft survival curves in relation to glomerular $\mathrm{C} 4 \mathrm{~d}$ staining and diagnosis of post-Tx IgAN Groups included were glomerular C4d positive post-Tx IgAN patients $(N=18$, black solid line), glomerular $\mathrm{C} 4 \mathrm{~d}$ negative post-Tx IgAN patients $(N=9$, grey dashed line) and patients without post-Tx GN $(N=801$, grey solid line). Log-Rank test showed significant allograftsurvival between these three groups $(p=0.01)$

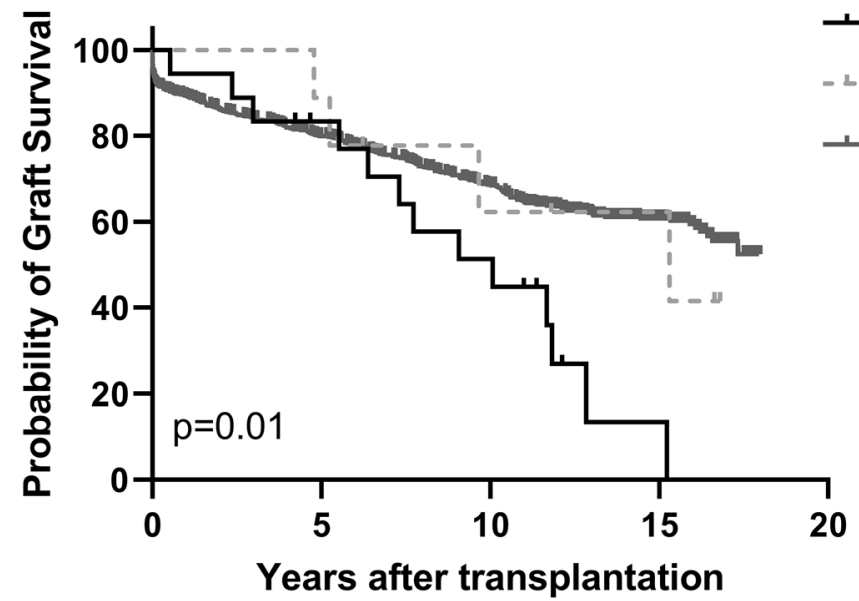

- C4d pos. post-Tx IgAN

- - C4d neg. post-Tx IgAN

- No post-Tx GN
Patients at risk

$\begin{array}{llll}18 & 14 & 9 & 2 \\ 9 & 9 & 5 & 4 \\ 801 & 552 & 396 & 121\end{array}$

Table 4 Cox regression model in relation to death-censored graft loss: univariate and multivariate analysis in all 883 patients

\begin{tabular}{|c|c|c|c|c|}
\hline \multirow[t]{2}{*}{ Parameters } & \multicolumn{2}{|l|}{ Univariate analysis } & \multicolumn{2}{|c|}{ Multivariate Analysis } \\
\hline & $\overline{\mathrm{HR}}(95 \% \mathrm{CI})$ & $\overline{P-v a l u e}$ & $\mathrm{HR}(95 \% \mathrm{CI})$ & $P$ value \\
\hline Reference: No glomerulonephritis & - & - & - & - \\
\hline C4dG positive post-Tx IgAN & $2.22(1.27-3.87)$ & 0.005 & $3.57(1.80-7.09)$ & 0.001 \\
\hline C4dG negative post-Tx IgAN & $1.15(0.43-3.08)$ & 0.79 & - & - \\
\hline Living Donor & $0.74(0.52-1.06)$ & 0.10 & - & - \\
\hline Re-transplantation & $1.66(1.29-2.13)$ & $<0.001$ & $1.10(0.69-1.74)$ & 0.69 \\
\hline HLA-mismatch, per number & $1.08(1.00-1.17)$ & 0.048 & $1.07(0.95-1.25)$ & 0.31 \\
\hline Sensitized patients & $1.54(1.21-1.97)$ & $<0.001$ & $1.46(0.99-2.15)$ & 0.059 \\
\hline Donor Age in years & $1.01(1.01-1.02)$ & 0.001 & $1.00(0.99-1.02)$ & 0.31 \\
\hline Cold ischemia time & $1.01(1.00-1.03)$ & 0.046 & $1.00(0.97-1.02)$ & 0.76 \\
\hline eGFR after 1 year, per $\mathrm{ml} / \mathrm{min} / 1.73 \mathrm{~m}^{2}$ & $0.95(0.95-0.96)$ & $<0.001$ & $0.97(0.96-0.98)$ & $<0.001$ \\
\hline Protein/creatinine quotient $>0.5 \mathrm{~g}$ after 1 year & $2.80(1.92-4.09)$ & $<0.001$ & $2.52(1.60-3.95)$ & $<0.001$ \\
\hline Early Banff Borderline & $0.62(0.44-0.88)$ & 0.008 & $0.83(0.50-1.37)$ & 0.46 \\
\hline Early TCMR Banff $\geq 1$ & $1.50(1.20-1.88)$ & $<0.001$ & $1.17(0.82-1.66)$ & 0.39 \\
\hline Early C4d positive ABMR & $1.78(1.32-2.38)$ & $<0.001$ & $1.16(0.78-1.74)$ & 0.47 \\
\hline Early C4d negative ABMR* & $2.03(1.47-2.80)$ & $<0.001$ & $2.73(1.73-4.31)$ & $<0.001$ \\
\hline Underlying renal disease IgAN & $0.90(0.57-1.44)$ & 0.67 & - & - \\
\hline Underlying renal disease diabetic nephropathy & $0.80(0.54-1.18)$ & 0.26 & - & - \\
\hline
\end{tabular}

$A B M R$ antibody-mediated rejection, GN Glomerulonephritis, post-Tx IgAN post-transplant IgA nephropathy; HLA human leukocyte antigen, $e G F R$ estimated glomerular filtration rate (Mayo Clinic formula). Early rejection was classified as occurrence within the first 6 months after transplantation, *: Suspicion of C4d negative ABMR, which consisted of the presence of at least two lesions being compatible with ABMR including a g score $>0$, a ptc score $>0$; post-transplant thrombotic microangiopathy (TMA), V3 lesions and a cg score $>0$ 
Author contributions Research idea and study design: ME, NK, ZK; Data acquisition: HO, CA, BP, RRS, JK, Data analysis/interpretation: ME, ZK, GB, GAB, JK; Statistical analysis: ZK, GAB; Supervision or mentorship: ZK, GAB

Funding Open access funding provided by Medical University of Vienna. NK is recipient of an Erwin Schrödinger Fellowship - Austrian Science Fund (FWF) (Project Nr. J-4377).

\section{Compliance with ethical standards}

Conflict of interest The authors declare no conflict of interest.

Ethical statement All studies in this review have been approved by the appropriate ethics committee and have therefore been performed in accordance with the ethical standards laid down in the 1964 Declaration of Helsinki and its later amendments.

Informed consent For this type of study, formal consent is not required.

Open Access This article is licensed under a Creative Commons Attribution 4.0 International License, which permits use, sharing, adaptation, distribution and reproduction in any medium or format, as long as you give appropriate credit to the original author(s) and the source, provide a link to the Creative Commons licence, and indicate if changes were made. The images or other third party material in this article are included in the article's Creative Commons licence, unless indicated otherwise in a credit line to the material. If material is not included in the article's Creative Commons licence and your intended use is not permitted by statutory regulation or exceeds the permitted use, you will need to obtain permission directly from the copyright holder. To view a copy of this licence, visit http://creativecommons.org/licenses/by/4.0/.

\section{References}

1. Jha V, Garcia-Garcia G, Iseki K, Li Z, Naicker S, Plattner B et al (2013) Chronic kidney disease: global dimension and perspectives. The Lancet 382(9888):260-272

2. D'Amico G (1987) The commonest glomerulonephritis in the world: IgA nephropathy. Q J Med 64(245):709-727

3. Le W, Liang S, Hu Y, Deng K, Bao H, Zeng C et al (2012) Longterm renal survival and related risk factors in patients with IgA nephropathy: results from a cohort of 1155 cases in a Chinese adult population. Nephrol Dial Transplant 27(4):1479-1485

4. Jarrick S, Lundberg S, Welander A, Carrero J-J, Höijer J, Bottai M et al (2019) Mortality in IgA Nephropathy: a Nationwide Population-Based Cohort Study. J Am Soc Nephrol 30(5):866-876

5. Moriyama T, Tanaka K, Iwasaki C, Oshima Y, Ochi A, Kataoka $\mathrm{H}$ et al (2014) Prognosis in IgA nephropathy: 30-year analysis of 1012 patients at a single center in Japan. PLoS ONE 9(3):e91756

6. Moldoveanu Z, Wyatt RJ, Lee JY, Tomana M, Julian BA, Mestecky J et al (2007) Patients with IgA nephropathy have increased serum galactose-deficient IgA1 levels. Kidney Int 71(11):1148-1154

7. Allen AC, Harper SJ, Feehally J (1995) Galactosylation of N- and O-linked carbohydrate moieties of IgA1 and IgG in IgA nephropathy. Clin Exp Immunol 100(3):470-474

8. Tomana M, Novak J, Julian BA, Matousovic K, Konecny K, Mestecky J (1999) Circulating immune complexes in IgA nephropathy consist of IgA1 with galactose-deficient hinge region and antiglycan antibodies. J Clin Invest 104(1):73-81

9. Suzuki H, Fan R, Zhang Z, Brown R, Hall S, Julian BA et al (2009) Aberrantly glycosylated IgA1 in IgA nephropathy patients is recognized by $\mathrm{IgG}$ antibodies with restricted heterogeneity. $\mathrm{J}$ Clin Invest 119(6): 1668-1677

10. Jennette JC (1988) The immunohistology of IgA nephropathy. Am J Kidney Dis 12(5):348-352

11. Miyazaki R, Kuroda M, Akiyama T, Otani I, Tofuku Y, Takeda $\mathrm{R}$ (1984) Glomerular deposition and serum levels of complement control proteins in patients with IgA nephropathy. Clin Nephrol 21(6):335-340

12. Maillard N, Wyatt RJ, Julian BA, Kiryluk K, Gharavi A, Fremeaux-Bacchi $\mathrm{V}$ et al (2015) Current understanding of the role of complement in IgA nephropathy. J Am Soc Nephrol 26(7):1503-1512

13. Roos A, Rastaldi MP, Calvaresi N, Oortwijn BD, Schlagwein N, van Gijlswijk-Janssen DJ et al (2006) Glomerular activation of the lectin pathway of complement in $\operatorname{IgA}$ nephropathy is associated with more severe renal disease. J Am Soc Nephrol 17(6): 1724-1734

14. Faria B, Canão $P$, Cai Q, Henriques C, Matos AC, Poppelaars F, et al. Arteriolar C4d in IgA Nephropathy: A Cohort Study. American Journal of Kidney Diseases. 2020

15. Faria B, Henriques C, Matos AC, Daha MR, Pestana M, Seelen M (2015) Combined C4d and CD3 immunostaining predicts immunoglobulin (Ig)A nephropathy progression. Clin Exp Immunol 179(2):354-361

16. Maeng YI, Kim MK, Park JB, Cho CH, Oh HK, Sung WJ et al (2013) Glomerular and tubular C4d depositions in IgA nephropathy: relations with histopathology and with albuminuria. Int J Clin Exp Pathol 6(5):904-910

17. Rath A, Tewari R, Mendonca S, Badwal S, Nijhawan VS (2016) Oxford classification of $\operatorname{IgA}$ nephropathy and $\mathrm{C} 4 \mathrm{~d}$ deposition; correlation and its implication. J Nephropharmacol 5(2):75-79

18. Sahin OZ, Yavas H, Tasli F, Gibyeli DG, Ersoy R, Uzum A et al (2014) Prognostic value of glomerular C4d staining in patients with IgA nephritis. Int J Clin Exp Pathol 7(6):3299-3304

19. Heybeli C, Unlu M, Yildiz S, Cavdar C, Sarioglu S, Camsari T (2015) IgA nephropathy: association of C4d with clinical and histopathological findings and possible role of IgM. Ren Fail 37(9):1464-1469

20. Drachenberg CB, Papadimitriou JC, Chandra P, Haririan A, Mendley S, Weir MR et al (2019) Epidemiology and Pathophysiology of Glomerular C4d Staining in Native Kidney Biopsies. Kidney Int Rep 4(11):1555-1567

21. Espinosa M, Ortega R, Sanchez M, Segarra A, Salcedo MT, Gonzalez F et al (2014) Association of C4d deposition with clinical outcomes in IgA nephropathy. Clin J Am Soc Nephrol 9(5):897-904

22. Segarra A, Romero K, Agraz I, Ramos N, Madrid A, Carnicer C et al (2018) Mesangial C4d Deposits in Early IgA Nephropathy. Clin J Am Soc Nephrol 13(2):258-264

23. Wagrowska-Danilewicz M, Danilewicz M (2017) The utility of glomerular $\mathrm{C} 4 \mathrm{~d}$ immunostaining in renal biopsies in patients with immunoglobulin A nephropathy. A clinicopathological study. Polish J Pathol 68(2):148-152

24. Kikic Z, Kozakowski N, Regele H, Priessner K, Nordmeyer V, Marinova L et al (2014) Clinicopathological relevance of granular $\mathrm{C} 4 \mathrm{~d}$ deposition in peritubular capillaries of kidney allografts. Transpl Int 27(3):312-321

25. Feucht HE (2003) Complement C4d in graft capillaries-the missing link in the recognition of humoral alloreactivity. Am J Transplant 3(6):646-652

26. Bohmig GA, Kikic Z, Wahrmann M, Eskandary F, Aliabadi AZ, Zlabinger GJ et al (2016) Detection of alloantibody-mediated complement activation: a diagnostic advance in monitoring kidney transplant rejection? Clin Biochem 49(4-5):394-403

27. Sapir-Pichhadze R, Curran SP, John R, Tricco AC, Uleryk E, Laupacis A et al (2015) A systematic review of the role of $\mathrm{C} 4 \mathrm{~d}$ 
in the diagnosis of acute antibody-mediated rejection. Kidney Int 87(1): 182-194

28. Berthelot L, Robert T, Vuiblet V, Tabary T, Braconnier A, Drame M et al (2015) Recurrent IgA nephropathy is predicted by altered glycosylated IgA, autoantibodies and soluble CD89 complexes. Kidney Int 88(4):815-822

29. Garnier AS, Duveau A, Demiselle J, Croue A, Subra JF, Sayegh J et al (2018) Early post-transplant serum IgA level is associated with IgA nephropathy recurrence after kidney transplantation. PLoS ONE 13(4):e0196101

30. Berthoux F, El Deeb S, Mariat C, Diconne E, Laurent B, Thibaudin L (2008) Antithymocyte globulin (ATG) induction therapy and disease recurrence in renal transplant recipients with primary IgA nephropathy. Transplantation 85(10):1505-1507

31. Regele H, Bohmig GA, Habicht A, Gollowitzer D, Schillinger M, Rockenschaub S et al (2002) Capillary deposition of complement split product $\mathrm{C} 4 \mathrm{~d}$ in renal allografts is associated with basement membrane injury in peritubular and glomerular capillaries: a contribution of humoral immunity to chronic allograft rejection. J Am Soc Nephrol 13(9):2371-2380

32. Chua JS, Zandbergen M, Wolterbeek R, Baelde HJ, van Es LA, de Fijter JW et al (2019) Complement-mediated microangiopathy in $\operatorname{Ig}$ A nephropathy and $\operatorname{IgA}$ vasculitis with nephritis. Mod Pathol 32(8):1147-1157

33. Rule AD, Larson TS, Bergstralh EJ, Slezak JM, Jacobsen SJ, Cosio FG (2004) Using serum creatinine to estimate glomerular filtration rate: accuracy in good health and in chronic kidney disease. Ann Intern Med 141(12):929-937

34. Network AWGotIIN, the Renal Pathology S, Roberts ISD, Cook HT, Troyanov S, Alpers CE et al (2009) The Oxford classification of IgA nephropathy: pathology definitions, correlations, and reproducibility. Kidney Int 76(5):546-556

35. Haas M, Loupy A, Lefaucheur C, Roufosse C, Glotz D, Seron D et al (2018) The Banff 2017 Kidney Meeting Report: revised diagnostic criteria for chronic active T cell-mediated rejection, antibody-mediated rejection, and prospects for integrative endpoints for next-generation clinical trials. Am J Transplant 18(2):293-307
36. Loupy A, Haas M, Solez K, Racusen L, Glotz D, Seron D et al (2017) The Banff 2015 Kidney Meeting Report: current challenges in rejection classification and prospects for adopting molecular pathology. Am J Transplant 17(1):28-41

37. Trimarchi H, Barratt J, Cattran DC, Cook HT, Coppo R, Haas M et al (2017) Oxford Classification of IgA nephropathy 2016: an update from the IgA Nephropathy Classification Working Group. Kidney Int 91(5):1014-1021

38. Kozakowski N, Herkner H, Eskandary F, Eder M, Winnicki W, Kläger J et al (2018) An integrative approach for the assessment of peritubular capillaritis extent and score in low-grade microvascular inflammation - associations with transplant glomerulopathy and graft loss. Nephrol Dial Transplant 34(1):166-174

39. Espinosa M, Ortega R, Gomez-Carrasco JM, Lopez-Rubio F, Lopez-Andreu M, Lopez-Oliva MO et al (2009) Mesangial C4d deposition: a new prognostic factor in IgA nephropathy. Nephrol Dial Transplant 24(3):886-891

40. Kusano T, Takano H, Kang D, Nagahama K, Aoki M, Morita $\mathrm{M}$ et al (2016) Endothelial cell injury in acute and chronic glomerular lesions in patients with IgA nephropathy. Hum Pathol 49:135-144

41. Chailimpamontree W, Dmitrienko S, Li G, Balshaw R, Magil A, Shapiro RJ et al (2009) Probability, predictors, and prognosis of posttransplantation glomerulonephritis. J Am Soc Nephrol 20(4):843-851

42. Regele H, Exner M, Watschinger B, Wenter C, Wahrmann M, Osterreicher C et al (2001) Endothelial C4d deposition is associated with inferior kidney allograft outcome independently of cellular rejection. Nephrol Dial Transplant 16(10):2058-2066

Publisher's Note Springer Nature remains neutral with regard to jurisdictional claims in published maps and institutional affiliations. 